\title{
Effects of Enterococcus faecalis CECT 7121 on Cryptosporidium parvum infection in mice
}

\author{
Valeria F. Del Coco ${ }^{1,2}$ - Mónica D. Sparo ${ }^{1}$ - Alicia Sidoti ${ }^{3}$ - Mónica Santín ${ }^{4}$. \\ Juan Angel Basualdo ${ }^{1}$ • María Alejandra Córdoba ${ }^{1,5}$
}

Received: 7 April 2016 / Accepted: 20 April 2016/Published online: 19 May 2016

(C) Springer-Verlag Berlin Heidelberg 2016

\begin{abstract}
Cryptosporidium is an opportunistic protozoan parasite of humans and animals worldwide and causes diarrheal disease that is typically self-limiting in immunocompetent hosts but often life threatening to immunocompromised individuals. However, there is a lack of completely efficient therapy available. Probiotics have attracted the attention as potential antiparasite compounds against protozoa involved in intestinal infections. This study investigated the effects of administration of probiotic Enterococcus faecalis CECT 7121 on Cryptosporidium parvum infection in immunosuppressed mice. Effects on C. parvum infection at the intestinal mucosa were studied and scored at each portion of the gut. It was demonstrated that $E f$ CECT 7121 interfered with C. parvum infection when both probiotic and parasite were present in the same intestinal location suggesting that $E f$ CECT 7121 supplementation can alleviate the negative effects of $C$. parvum infection.
\end{abstract}

Valeria F. Del Coco

valeriadelcoco@med.unlp.edu.ar

1 Centro de Estudios Microbiológicos y Parasitológicos (CUDEMyP), Facultad de Ciencias Médicas, Universidad Nacional de La Plata, 60 y 120 , La Plata 1900 , Argentina

2 Consejo Nacional de Investigaciones Científicas y Técnicas (CONICET), Buenos Aires, Argentina

3 Cátedra de Patología B, Facultad de Ciencias Médicas, Universidad Nacional de La Plata, La Plata, Buenos Aires, Argentina

4 Environmental Microbial and Food Safety Laboratory, Beltsville Agricultural Research Center, United States Department of Agriculture, Beltsville, MD, USA

5 Comisión de Investigaciones Científicas de la Provincia de Buenos Aires, La Plata, Buenos Aires, Argentina
Keywords Cryptosporidium • Probiotics . Immunosuppressed mice $\cdot$ Enterococcus faecalis CECT7121

\section{Introduction}

Cryptosporidium parvum is a zoonotic protozoan parasite recognized worldwide as an important public health concern, which can be life threatening in children and patients with a poor T CD4 ${ }^{+}$immune response (Del Coco et al. 2009). In the absence of an effective therapy for cryptosporidiosis, more than 200 antimicrobial agents were tested against Cryptosporidium but none have been clearly efficacious (Rossignol 2010). An effective treatment against Cryptosporidium would benefit immunocompetent hosts with self-limited diarrhea and severely immunocompromised patients that suffer chronic infection (Stockdale et al. 2008).

Probiotics used as a microbial interference therapy offer an attractive alternative for control gastrointestinal infections alone or as an adjuvant of the main antimicrobial therapy (Van Niel et al. 2002; Benyacoub et al. 2005; Goyal et al. 2011). There are few studies that evaluated the effect of probiotics on parasitic infections (Travers et al. 2011). Oral administration of Lactobacillus rhamnosus GG in Giardia duodenalis-infected BALB/c mice reduced both the severity and duration of giardiasis (Goyal et al. 2013). Enterococcus faecium SF68 and Lactobacillus johnsonii La1 were also effective in eliminating Giardia infection from mice and gerbils, respectively (Benyacoub et al. 2005; Humen et al. 2005). A reduction of recovered Toxocara canis larvae at different tissues was observed after the administration of Saccharomyces boulardii in mice (da Costa de Avila et al. 2012). For Cryptosporidium, beneficial effects of the use of different strains of Lactobacillus against C. parvum have been demonstrated (Waters et al. 1999; Alak et al. 1997, 
1999). The colonization with Lactobacillus reuteri of gnotobiotic $\mathrm{T}$ cell receptor (TCR)-alpha-mice decreased the numbers of $C$. parvum detected in intestinal tissues (Waters et al. 1999). A decreased of C. parvum oocyst shedding was also observed in C57BL/6 female mice immunosuppressed by murine leukemia virus fed daily with $L$. reuteri or Lactobacillus acidophilus (Alak et al. 1997, 1999). Because the biological properties of probiotics are species and strain specific, testing of additional probiotic microorganisms against $C$. parvum infection appears warranted.

Enterococcus faecalis is a nonmotile, gram-positive, spherical bacterium, formerly classified as part of the Lancefield group D Streptococcus and one of the most prevalent species isolated from humans. The E. faecalis CECT 7121 ( $E f$ CECT7121) strain was recovered from natural corn silage in Buenos Aires, Argentina (European patent EP1816190; US Patent Application 20080063666 Kind Code: A1). Ef CECT7121 does not express virulence factors such as hemolysin or gelatinase and lacks a capsule. It is resistant to gastric $\mathrm{pH}$ and bile and has not shown antimicrobial resistance (Sparo et al. 2006, 2008). It is of interest as a potential anti-C. parvum agent because it has already demonstrated biological activity against $T$. canis (Basualdo et al. 2007) and Salmonella enterica serovar Enteritidis in vivo (Sparo and Sanchez Bruni 2011). Therefore, the present study was undertaken to evaluate the effects of the administration of E. faecalis CETC 7121 on C. parvum IIaA21G1R1 infection in immunosuppressed mice.

\section{Materials and methods}

\section{Parasite}

Fresh Cryptosporidium oocysts were obtained from a calf on a dairy farm in the province of Buenos Aires, Argentina. Oocysts were concentrated by water ether technique (Bukhari and Smith 1995), purified by discontinuous sucrose density centrifugation (Heyman et al. 1986), and then counted with a Neubauer hemocytometer. A combination of streptomycin, penicillin, and amphotericin B (Gibco, Grand Island, $\mathrm{NY}$ ) was added and the suspension was stored at $4{ }^{\circ} \mathrm{C}$. Cryptosporidium, in this study, was identified by a nested PCR protocol to amplify an 830-bp fragment of the small subunit ribosomal RNA (SSU rRNA) gene (Santín et al. 2008) followed by a nested PCR that amplified a fragment of the GP60 gene (Sulaiman et al. 2005). The SSU rRNA nucleotide sequence had a $100 \%$ similarity with $C$. parvum (GenBank accession number AF093493), and the GP60 nucleotide sequence had a $100 \%$ similarity with $C$. parvum subtype IIaA21G1R1 (GenBank accession number JQ861957).
Mice included in groups requiring $C$. parvum infection were inoculated orally at day 8 from the beginning of the experiment with $10^{5}$ oocysts of $C$. parvum IIaA21G1R1 as described (Del Coco et al. 2012).

\section{Bacterial strain, preparation, and inoculation}

Bacterial suspension was prepared from a $10-\mathrm{ml}$ culture of $E f$ CECT7121 propagated in $100 \mathrm{ml}$ of brain heart infusion broth (BHI, Britania, Buenos Aires, Argentina) at $35^{\circ} \mathrm{C}$ for $18 \mathrm{~h}$ (Sparo et al. 2006). After incubation, the suspension was centrifuged for $15 \mathrm{~min}$ at $6000 \times \mathrm{g}$ and washed three times with sterile physiologic solution (PS) using the same centrifugation protocol before preparing the suspension in BHI medium, at a final concentration of $10^{8}$ colony forming units $/ \mathrm{ml}(\mathrm{CFU} / \mathrm{ml})$. Administration of Ef CECT $7121\left(2 \times 10^{7}\right.$ UFC) was performed before, during, and after the experimental infection with $C$. parvum oocysts on days $6,7,8,9$, and 10 from the beginning of the experiment.

\section{Animals}

The mouse model used in this study has been previously described (Del Coco et al. 2012). Male N: NIH Swiss mice, 3 weeks of age, were obtained from the Department of Animal Laboratory Sciences, School of Veterinary Sciences, University of La Plata, Buenos Aires, Argentina. Mice were housed individually in plastic cages with wire mesh tops, under pathogen-free conditions, and kept on a 12-h cycle of light and dark. Mice received sterilized food and water. Animal handling and all experimental procedures were carried out in compliance with the "Guide for the Care and Use of Laboratory Animals" (National Research Council 1996). The experimental protocol was approved by the Animal Welfare Committee of the School of Medical Sciences, University of La Plata.

\section{Groups of animals}

Mice in groups requiring immunosuppression were treated throughout the experiment (43 days) with a daily dose of $100 \mu \mathrm{g}$ of dexamethasone sodium phosphate in drinking water.

One hundred and five mice were divided into 7 groups of 15 animals each. Mice in group I were immunosuppressed, infected with $C$. parvum IIaA21G1R1, and treated with $E f$ CECT 7121. The following six control groups (II-VII) were also included: immunosuppressed mice infected with $C$. parvum, untreated with $E f$ CECT 7121 (group II); immunosuppressed uninfected mice, treated with $E f$ CECT 7121 (group III); immunocompetent uninfected mice, treated with EfCECT 7121 (group IV); immunocompetent mice inoculated with $C$. parvum oocysts, untreated with $E f$ CECT 7121 
(group V); immunocompetent, uninfected mice, untreated (group VI); and immunosuppressed uninfected mice, untreated (group VII).

At days of 7, 14, 21, 28, and 35 post infection (p.i.), three mice per group were euthanized and organs removed.

\section{Histological examination}

Tissue samples harvested from the duodenum, jejunum, ileum, cecum, large intestine, lungs, liver, gall bladder, and pancreas were fixed in $10 \%$ formalin, embedded in paraffin, sectioned, stained with hematoxylin and eosin ( $\mathrm{H} \& \mathrm{E})$, and observed microscopically at $\times 400$ magnification. Cryptosporidial infection at the intestinal mucosa was scored as described by Del Coco et al. (2012).

\section{Ef CECT 7121 detection}

Samples from each portion of the intestine were analyzed to evaluate the presence of Ef CECT7121. The spleen was removed to determine translocation of Ef CECT7121. Samples were taken aseptically, placed in sterile tubes, sonicated for $2 \mathrm{~min}$, and subsequently centrifuged at $700 \times g$ for $1 \mathrm{~min}$. Supernatants were aspirated centrifuged at $9000 \times \mathrm{g}$ and then cultivated in bile esculin azide agar (Britania, Buenos Aires, Argentina) at $35{ }^{\circ} \mathrm{C}$ for $24 \mathrm{~h}$ to detect viable enterococci (Sparo et al. 2006). Ef CECT7121 colonies were confirmed by phenotypical characterization (Sparo and Sanchez Bruni 2011).

Additionally, a whole cell protein profile (WCP) was obtained (Merquior et al. 1994) and compared to the reference strain E. faecalis ATCC 29212. A densitometric analysis was carried out using Image Pro and Origin 6.0 software (Germany). The homology percentage was calculated using the Dice's coefficient (Sparo and Sanchez Bruni 2011). Finally, total DNA was extracted according to Persing et al. (2008) and randomly amplified polymorphic DNA (RAPD) technique was employed to confirm the identity of Ef CECT7121, using primers D8635 (5 'CGG CCA GAG AAG GCA GGA GAC 3') and M13 (5 'GAG GGT GGC GGT TCT 3') (Suzzi et al. 2000). Results were analyzed by RAPDistance 1.04 software package (Australian National University).

\section{Statistical analysis}

Statistical differences were determined using one-way and multifactorial ANOVA, followed by pairwise comparison by Tukey's or Bonferroni's tests as appropriate. To evaluate infection at the mucosa, the dependent variable was score of infection; days p.i. and sections of intestine were the independent variables. A full model multifactorial
ANOVA analysis was performed, with a subsequent oneway ANOVA performed as the reduced model. Frequency measurements and simple regression were also applied. Significance was established at $p<0.05$ (Stat-graphics Centurion XV for Windows, version 15.02, 2005).

\section{Results}

No animals died during the experiment.

\section{Detection of $E f$ CECT7121}

Ef CECT7121 was identified at the intestine of mice inoculated with this strain (groups I, III, and IV) and was not detected at the spleen. In all treated mice, $E f$ CECT7121 was identified from the ileum to the colon on days 7, 14, 21, and 28 days p.i. but not at 35 days p.i. The duodenum and jejunum showed no colonization.

\section{Histological examination}

C. parvum was found at the microvillus border of duodenum, proximal and distal jejunum, and ileum of mice in groups I and II. Mice from group I exhibited the lowest average score of infection without statistical significance $(p=0.3116)$. Parasites were not found in the colon or extra intestinal sites, or in mice in any other groups.

In group I, $47 \%$ of mice $(7 / 15)$ were infected in the duodenum, $40 \%(6 / 15)$ in the proximal jejunum, $13 \%(2 / 15)$ in the distal jejunum, and $20 \%(3 / 15)$ in the ileum. Mice from group II had the overall highest rate of infection; $40 \%$ of mice $(6 / 15)$ were infected in the duodenum, $60 \%(9 / 15)$ in the proximal jejunum, $60 \%(9 / 15)$ in the distal jejunum, and $60 \%(9 / 15)$ in the ileum.

The intensity of infection in the different portions at each site within the intestine was compared for groups I and II (the only two groups in which $C$. parvum was found) $(p=0.28)$. Mice in group I had the most organisms per site in the duodenum, ileum, and proximal jejunum. Mice in group II had the most organisms persisted in the ileum, distal jejunum, and duodenum (Fig. 1). A considerable reduction of $C$. parvum infection in mice treated with Ef CECT7121 was seen on day 21 p.i. $(p=0.0205)$. Parasite load decreased drastically from intestinal epithelium on day 28 p.i., increasing the score of infection on day 35 p.i. in both groups (Fig. 2). This is in concordance with the decrement of the probiotic at the intestine on day 28 p.i. and its complete depuration on day 35 p.i. (data not shown). 
Fig. 1 Average of score of infection at different portions of the intestine from N: NIH Swiss immunosuppressed C. parvuminfected mice that received or not treatment with $E f$ CECT7121

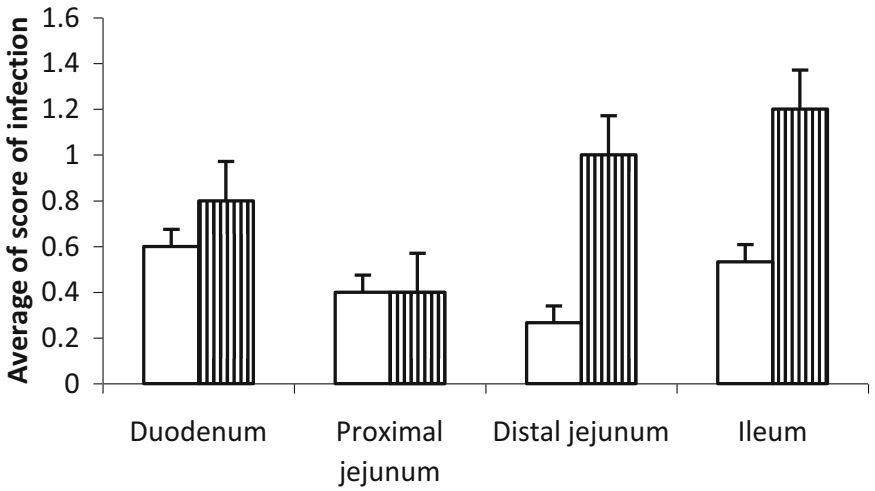

口Group I (C. parvum + Ef CECT 7121)

m Group II (C. parvum)

\section{Discussion}

In the present work, the effects of $E f \mathrm{CECT} 7121$ on C. parvum IIaA21G1R1 infection were studied in a mouse model of immunosuppression using dexamethasone. This is the first study in which the interaction between a pathogen and a probiotic is evaluated at each portion of the gut. It is important to know the location of each microorganism, in our case parasite C. parvum and probiotic Ef CECT7121, because they must share the place of colonization if the goal is to produce an interference effect. It is important to establish a correlation between the location of the probiotic strain and its biological effects (Pavan et al. 2003). EfCECT7121 was detected at the ileum and colon of treated mice (groups I, II, and IV).

Although Ef CECT7121 was not able to eradicate or prevent $C$. parvum infection in treated mice, the average infection score was lower than for infected untreated mice. Only 3 of 15 infected animals supplemented with Ef CECT7121 and infected with C. parvum (group I) had parasites in the ileum, the site with the highest score of infection in infected and untreated mice (9/15) (group II). This circumstance could be related to the pattern of intestinal colonization of $E f$ CECT7121 as indicated by the fact that the duodenum and proximal jejunum were the most affected portions of the gut in mice infected with C. parvum and treated with Ef CET7121 (group I) and where the probiotic was undetectable.
The effect of $E f$ CECT7121 on $C$. parvum infection was particularly significant at day 21 p.i. when infected and untreated mice (group II) presented a peak of infection whereas infected and treated mice (group I) presented a significant reduction of the infection score. The increase of the score of infection at day 35 p.i. could be related to the clearance of the probiotic at the intestine, which could have allowed the reinfection by autoinfective stages of $C$. parvum. This fact suggests that $E f$ CECT7121 should be administered for a longer period to effectible control chronic cryptosporidiosis.

The safety of $E f C E C T 7121$ was established by the absence of $E f C E C T 7121$ in the spleen in all treated mice (groups I, III, and IV) which confirmed its inability to move to colonize extraintestinal organs via circulatory system.

The mechanisms underlying the beneficial effect of the probiotic $E f$ CECT7121 on C. parvum infection is unknown and could be attributed to several factors: competition for binding sites on the gut epithelium (Del Coco 2015); the antimicrobial effect of the peptide secreted by Ef CECT 7121 which may adversely affect the survival of microorganisms (Sparo et al. 2006); or changes in the microenvironment generated by the release of metabolic products of the probiotic (Oelschlaeger 2010). An important fact is that Cryptosporidium needs an alkaline environment to excyst (Smith et al. 2005), and the acidification of the medium induced by this lactic acid bacteria could affect this process reducing its viability.
Fig. 2 Average of score of infection at different times post infection observed at the intestine from N: NIH Swiss immunosuppressed C. parvuminfected mice that received or not treatment with $E f$ CECT7121. ${ }^{*} p<0.05$

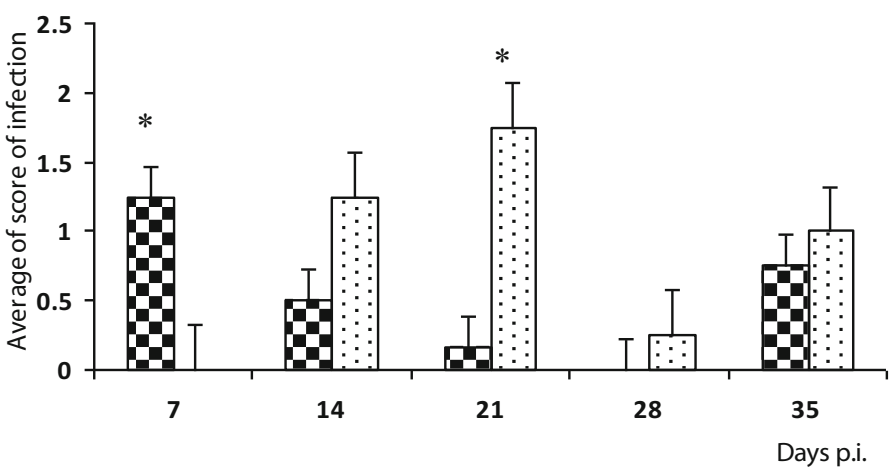
CECT 7121) $\checkmark$ Group II (C. parvum) 
Modulation of immune system constitutes other important issue. In the gut, this strain could interact with the Peyer's patches $\mathrm{M}$ cells and immune cells and takes place an increase in the number of IgA-producing cells, production of IgM, and secretory IgA (Adams 2010).

A previous study tested the administration at three different doses of $E f$ CECT 7121 to control T. canis infection in mice observing an important reduction of T. canis infection in those mice (Basualdo et al. 2007).

There are few studies that have evaluated the effects of probiotics on parasitic infections. Alak et al. (1997) have studied the effect of $L$. reuteri on $C$. parvum infection in a murine model of acquired immunodeficiency syndrome. They administered 26 doses of $1 \times 10^{8}$ UFC of $L$. reuteri, 10 days before and 16 days after the experimental infection with C. parvum, and observed a considerable reduction of $C$. parvum infection revealed by the histology of the stomach, distal ileum, and colon. In contrast, Guitard et al. (2006) did not find an anticryptosporidial effect using two commercial preparations: Actimel ${ }^{\circledR}$ (L. casei, L. Bulgaricus, Streptococcus thermophilus) and VSL\#3 ${ }^{\circledR}$ (L. casei, L. bulgaricus, Lactobacillus plantarum, L. acidophilus, Bifidobacterium infantis, Bifidobacterium breve, S. thermophilus) both administered during 21 days (before, during, and after inoculation with $C$. parvum oocysts) in neonatal rats.

In the present study, $E f$ CECT 7121 demonstrated interference in vivo against $C$. parvum where both probiotic and parasite were present at the same intestinal location. These satisfactory effects induced by the administration of $E f$ CECT 7121 on mice infected with C. parvum recommends further testing in combination with other probiotics or antiparasitic drugs against cryptosporidiosis.

Acknowledgments This work was supported by Universidad Nacional de La Plata.

\section{Compliance with ethical standards}

Conflict of interest The authors declare that they have no conflict of interest.

Ethical approval All applicable international, national, and/or institutional guidelines for the care and use of animals were followed.

\section{References}

Adams CA (2010) The probiotic paradox: live and dead cells are biological response modifiers. Nutr Res Rev 23:37-46

Alak JI, Wolf BW, Mdurvwa EG et al (1997) Effect of Lactobacillus reuteri on intestinal resistance to Cryptosporidium parvum infection in murine model of immunodeficiency syndrome. J Infect Dis 175: 218-221

Alak JI, Wolf BW, Mdurvwa EG, Pimentel et al (1999) Supplementation with Lactobacillus reuteri or L. Acidophilus reduced intestinal shedding of Cryptosporidium parvum oocysts in immunodeficient C57BL/6mice. Cell Mol Biol (Noisy-le-grand) 45:855-863

Basualdo J, Sparo M, Chiodo P et al (2007) Oral treatment with a potential probiotic (Enterococcus faecalis CECT7121) appears to reduce the parasite burden of mice infected with Toxocara canis. Ann Trop Med Parasitol 101:559-562

Benyacoub J, Pérez PF, Rochat F et al (2005) Enterococcus faecium SF68 enhances the immune response to Giardia intestinalis in mice. J Nutr 135:1171-1176

Bukhari Z, Smith HV (1995) Effect of three concentration techniques on viability of Cryptosporidium parvum oocysts recovered from bovine feces. J Clin Microbiol 33:2592-2595

de Avila LF, Conceição FR, Telmo PL et al (2012) Saccharomyces boulardii reduces infection intensity of mice with toxocariasis. Vet Parasitol 187:337-340

Del Coco VF (2015) Microorganisms conferring beneficial health effects. Rev Argent Microbiol 47:171-173

Del Coco VF, Córdoba MA, Basualdo JA (2009) Cryptosporidiosis: an emerging zoonosis. Rev Argent Microbiol 41:185-196

Del Coco VF, Córdoba MA, Sidoti A et al (2012) Experimental infection with Cryptosporidium parvum IIaA21G1R1 subtype in immunosuppressed mice. Vet Parasitol 190:411-417

Goyal N, Tiwari RP, Shukla G (2011) Lactobacillus rhamnosus GG as an effective probiotic for murine giardiasis. Interdiscip Perspect Infect Dis 795219

Goyal N, Rishi P, Shukla G (2013) Lactobacillus rhamnosus GG antagonizes Giardia intestinalis induced oxidative stress and intestinal disaccharidases: an experimental study. World J Microbiol Biotechnol 29:1049-1057

Guitard J, Menotti J, Desveaux A (2006) Experimental study of the effects of probiotics on Cryptosporidium parvum infection in neonatal rats. Parasitol Res 99:522-527

Heyman MB, Shigekuni LK, Ammann AJ (1986) Separation of Cryptosporidium oocysts from fecal debris by density gradient centrifugation and glass bead columns. J Clin Microbiol 23:789-791

Humen MA, De Antoni GL, Benyacoub J (2005) Lactobacillus johnsonii La1 antagonizes Giardia intestinalis in vivo. Infect Immun 73:12651269

Merquior VLC, Peralta JM, Facklam RR et al (1994) Analysis of electrophoretic whole-cell protein profiles as a tool for characterization of Enterococcus species. Curr Microbiol 28:149-153

National Research Council (1996) Guide for the care and use of laboratory animals Institute of Laboratory Animal Resources. National Academy Press, Washington DC

Oelschlaeger TA (2010) Mechanisms of probiotic actions - a review. Int J Med Microbiol 300:57-62

Pavan S, Desreumaux P, Mercenier A (2003) Use of mouse models to evaluate the persistence, safety, and immune modulation capacities of lactic acid bacteria. Clin Diagn Lab Immunol 10:696-701

Persing DH, Smith TF, Tanover FC (2008) Diagnostic molecular biology, principles and application. ASM Press, Washington DC

Rossignol JF (2010) Cryptosporidium and Giardia: treatment options and prospects for new drugs. Exp Parasitol 124:45-53

Santín M, Trout JM, Fayer R (2008) A longitudinal study of cryptosporidiosis in dairy cattle from birth to 2 years of age. Vet Parasitol 155: $15-23$

Smith HV, Nichols RAB, Grimason AM (2005) Cryptosporidium excystation and invasion: getting the guts of the matter. Trends Parasitol 21:133-142

Sparo M, Sanchez Bruni S (2011) Novel immune pharmacological approaches for the treatment of bacterial invasive infections. In: Mendez-Vilas A (ed) Science against microbial pathogens: communicating current research and technological advances, 1 st edn. Formatex, Badajoz, pp 135-143 
Sparo MD, Castro MS, Andino PJ et al (2006) Partial characterization of enterocin MR99 from a corn silage isolated of Enterococcus faecalis. J Appl Microbiol 100:123-134

Sparo M, Nuñez GG, Castro M et al (2008) Characteristics of an environmental strain, Enterococcus faecalis CECT7121, and its effects as additive on craft dry-fermented sausages. Food Microbiol 25: $607-615$

Stockdale HD, Spencer J, Blagburn B (2008) Prophylaxis and chemotherapy. In: Fayer R, Xiao L (eds) Cryptosporidium and cryptosporidiosis, 2nd edn. CRC Press, New York, pp 255-287

Sulaiman IM, Hira PR, Zhou L et al (2005) Unique endemicity of cryptosporidiosis in children in Kuwait. J Clin Microbiol 43:2805-2809
Suzzi G, Caruso M, Gardini F et al (2000) A survey of the enterococci isolated from an artisanal Italian goat's cheese (semicotto caprino). J Appl Microbiol 89:267-274

Travers MA, Florent I, Kohl L (2011) Probiotics for the control of parasites: an overview. J Parasitol Res 610769

Van Niel CW, Feudtner C, Garrison MM et al (2002) Lactobacillus therapy for acute infectious diarrhea in children: a meta-analysis. Pediatrics 109:678-684

Waters WR, Harp JA, Wannemuehler MJ et al (1999) Effects of Lactobacillus reuteri on Cryptosporidium parvum infection of gnotobiotic TCR-a-deficient mice. J Eukaryot Microbiol 46:60S-61S 